\title{
The Long-Lasting Struggle to Achieve Atomic-Resolution Microscopy by Correcting the Aberrations of Electron Lenses
}

\author{
Harald Rose
}

\section{Ulm University, Center for Electron Microscopy, Albert-Einstein-Allee 11, 89069 Ulm, Germany}

The correction of the aberrations of electron lenses is the long story of many seemingly fruitless efforts to improve the resolution of electron microscopes by compensating for aberrations of round electron lenses over a period of 50 years. The problem started in 1936 when Scherzer [1] demonstrated that the chromatic and spherical aberrations of rotationally symmetric electron lenses are unavoidable. Moreover, the coefficients of these aberrations cannot be made sufficiently small. As a result, the resolution limit of standard electron microscopes equals about one hundred times the wavelength of the electrons, whereas modern light microscopes have reached a resolution limit somewhat smaller than the wavelength. In 1947, Scherzer found an ingenious way for enabling aberration correction. He demonstrated in a famous article that it is in theory possible to eliminate chromatic and spherical aberrations by lifting any one of the constraints of his theorem, either by abandoning rotational symmetry or by introducing time-varying fields, or space charges [2]. Moreover, he proposed a multipole corrector compensating for the spherical aberration of the objective lens.

Starting in 1948, Seeliger built and tested the Scherzer corrector during a period of about five years. He aligned the constituent elements mechanically by means of adjustment screws. However, the experiments showed that this approach was a major obstacle due to insufficient stability of the mechanical alignment. As a result, the correction did not improve the resolution of the microscope because it was limited by mechanical and electromagnetic instabilities rather than by the static defects of the objective lens. Although Seeliger could not improve the resolution limit of the basic electron microscope, he could demonstrate that the corrector provided a negative spherical aberration, which could be adjusted to compensate for the spherical aberration of the objective lens [3]. In 1956, G. Moellenstedt first demonstrated experimentally the effective correction of spherical aberration by means of this corrector [4]. By employing critical illumination with a large cone angle of $2 \times 10^{-2} \mathrm{rad}$, he enlarged the spherical aberration to such an extent that it became by far the dominant aberration, which strongly blurred the image. After compensating for the spherical aberration by means of the octopoles, the resolution improved by a factor of about seven accompanied by a striking increase in contrast.

In 1964, Deltrap built a telescopic quadrupole-octopole corrector to eliminate the spherical aberration of a probe-forming lens. Although he nullified this aberration, he failed, like his predecessors, to improve the actual resolution of the uncorrected system because at that time the resolution was not limited by the spherical aberration of the objective lens. Moreover, all correctors known at that time introduce large off-axial coma and are not suitable for transmission electron microscopes (TEMs). In order to compensate for spherical aberration, chromatic aberration and off-axial coma, a novel aplanatic corrector utilizing symmetry properties was proposed [5]. This corrector was built and tested successfully in a test microscope within the frame of the so-called Darmstadt project and it demonstrated for the first time the simultaneous correction of chromatic and spherical aberrations [6]. Unfortunately, the project was abandoned after the death of O. Scherzer in 1982, although it was successful as far as it went. 
In 1972, A. Crewe and V. Beck started at the University of Chicago another attempt to correct the spherical aberration of a scanning transmission electron microscope (STEM). They built and tested over a period of six years a magnetic quadrupole-octopole corrector consisting of a symmetric quadruplet with combined quadrupoles and octopoles. Because the corrector is aimed for the STEM, the difficulties to simultaneously compensate for the off-axial coma did not arise. Although Beck and Crewe incorporated several stigmator coils for producing weak dipole and hexapole fields, they were unable to find a suitable setting. Although all fruitless experimental attempts demonstrated that correction works in principle, none of them achieved an improvement in resolution. The main reasons for theses failures were: (a) the basic microscope was not stable enough, (b) the deleterious interference with the environment had been underestimated, (c) it was not possible to determine the state of alignment with the required precision, and (d) the resolution-limiting residual aberrations could not be measured with the required accuracy and eliminated within a period of time which must be shorter than the duration of the overall stability of the entire system. These obstacles were so severe that worldwide the granting agencies refused to fund the "fruitless correction projects" any longer.

In 1990, the required technology and a new corrector design consisting of two sextupoles and two round lenses became available. Because this corrector is rather simple, Haider, Urban and the author were convinced that the successful correction of the spherical aberration in a $200 \mathrm{kV}$ TEM equipped with a field emission gun should be feasible. Fortunately, the Volkswagen foundation was willing to take the risk and approved funding in 1991. In July 1997, the first atomic-resolution images of gallium-arsenide were obtained after the resolution limit of the microscope was reduced by the corrector from $0.24 \mathrm{~nm}$ to about 0.12nm [7]. Around 1995 O. Krivanek developed at the Cavendish Laboratory in Cambridge an improved version of the Deltrap quadrupole octopole corrector which he incoporated in his $100 \mathrm{kV}$ VG STEM. With this corrector he achieved, in 1998, the first genuine improvement of the resolution of a STEM [8]. Owing to the unexpected success of these correction efforts, the funding situation improved considerably. In the US the Department of Energy (DOE) approved in 2003 funding of the TEAM (Transmission Electron Aberration-Corrected Microscope) project headed by U. Dahmen (LBNL Berkeley). The required resolution limit of $0.5 \AA$ was obtained in 2009 by means of an improved version of the hexapole corrector after the information limit of the basic $300 \mathrm{kV}$ TEM was lowered to about $0.4 \AA[9]$.

References:

[1] O Scherzer, Z. Physik 101 (1936), p. 593.

[2] O Scherzer, Optik 101 (1947), p. 114.

[3] R Seeliger, Optik 8 (1951), p. 311.

[4] G Moellenstedt, Optik 13 (1956), p, 209.

[5] H Rose, Optik 34 (1971), p. 285.

[6] H Koops, G Kuck and O Scherzer, Optik 48 (1977), p. 225.

[7] M Haider, H Rose, S Uhlemann, E Schwan, B Kabius and K Urban, J. Electron Microscopy 47 (1998), p. 395.

[8] O L Krivanek, N Dellby and A R Lupini, Ultramicroscopy 78 (1999), p.1.

[9] C Kisielowski et al., Microscopy and Microanalysis 14 (2008), p.469. 